Sharif University of Technology
Scientia Iranica
Transactions E: Industrial Engineering
http://scientiairanica.sharif.edu

\title{
A class Hotelling model for sequential auctions of close substitutes
}

\author{
E. $\mathrm{Hu}^{\mathrm{a}, \mathrm{b}}, \mathrm{C} \cdot \mathrm{Rao}^{\mathrm{c}, *}$, and Y. Zhao ${ }^{\mathrm{a}}$ \\ a. Systems Engineering Institute of Automation School, Huazhong University of Science and Technology, Wuhan 430074, P. R. \\ China. \\ b. School of Science, Hubei University of Technology, Wuhan 430068, P. R. China. \\ c. School of Science, Wuhan University of Technology, Wuhan 430070, P. R. China.
}

Received 10 September 2016; accepted 26 May 2018

\section{KEYWORDS}

Sequential auctions; Hotelling model;

Supply chain;

Associated valuation;

Information policy.

\begin{abstract}
Against the background of supply chains, this paper constructs a class Hotelling model to describe and explore sequential auctions of close substitutes with slightly more general associated valuations. In this generalized model, both close substitutes and bidders are hypothetically distributed at the interval $[0,1]$, types of bidders are continuous, and each bidder's valuations for close substitutes are not independent. Moreover, with the aid of this model, equilibriums are explored, and efficiencies of the auctions are analyzed under second-price sealed-bid auction formats. Further, considering two typical information policies, we investigate some concrete bids and revenues of the efficient sequential auctions, while bidders' valuations are linear functions of distances between them and close substitutes. Results show that efficiencies of the sequential auctions are conditional, and influences of information policies on revenues of the auctions are related to both numbers of bidders and locations of items.
\end{abstract}

(C) 2019 Sharif University of Technology. All rights reserved.

\section{Introduction}

In 1929, Hotelling developed a model of spatial competitions to demonstrate relationships between locations and pricing behaviors of firms through a line of fixed length and predict an aggregation of two competing firms in the middle of the customers' support interval $[1,2]$. The standard Hotelling model assumes that all consumers are identical (except for locations) and evenly dispersed along the line; both the firms and consumers respond to changes in demand and the economic environment. As a game model, it can also be used to describe some auction problems in supply chains: $m$ suppliers sell their supply contracts

*. Corresponding author.

E-mail address: cjrao@163.com (C.Rao)

doi: $10.24200 /$ sci. 2018.20546 sequentially or simultaneously to $n$ agents with unitdemands via auctions, and both suppliers and agents are located in the same traffic line. Here, suppliers and agents correspond to firms and customers, respectively. In addition, these contracts are deterministic and undifferentiated for agents; thus, differences among agents' valuations for contracts mainly depend on their transportation costs.

The above-mentioned problem can be abstracted as sequential auctions of close substitutes. Since the distance between an agent and his or her suppliers is considered, each agent's valuations for the suppliers' contracts are also considered, which are different from interdependent valuations among agents [3]. Hence, by means of the Hotelling model, our paper tries to focus on sequential auctions of close substitutes with slightly more general associated valuations. Here, key functions of close substitutes are the same or similar; however, their configurations or external performances 
are slightly different so that various needs of consumers are met; thus, the private valuations of consumers are not exactly the same.

A key consideration of sequential auctions is a bidder's expected surplus for the follow-up auctions, which is usually concerned with future objects, bidder numbers, previous winning information, etc. Thus, information policy is always an important topic for auctions or other business activities, and theoretical and experimental studies have shown that revealing some information in advance will possibly affect the overall efficiency and revenues of auctions [4,5]. Presently, there are major controversies and, consequently, a large body of literature about sequential auctions and information policies. Usually, while auctioned objects are heterogeneous and bidders' valuations are independent of each other, an expected revenue-maximizing auctioneer should fully and publicly reveal all information about auctioned objects [6-8]. However, while auctioned objects are homogeneous and bidders' valuations are not mutually independent, it was revealed that future objects or other related information in advance would uncertainly affect the overall efficiency and revenues of sequential auctions [9-12]. Currently, in these pieces of literature, a bidder's valuations for homogeneous objects are usually supposed to be identical or proportional, which may be considered as especially associated valuations and lead to learning behaviors [13]. Owing to the difficulty of modeling general associated valuations and processing mathematical expectations while a bidder type is multidimensional and continuous, existing researches mostly focus on sequential auctions where a bidder type is hypothetically discrete, namely $H$ (High) or $L$ (Low), and his or her valuations of objects are identical.

Recently, Zeithammer [14] studied sequential auctions of heterogeneous objects, discussed the influence of revealing future objects on auction efficiency and proved the existence of symmetric equilibriums and pure bidding strategies. Bayesian methods and the models used for processing a multi-dimensional continuous type in his paper inspired our studies. Accordingly, combined with characteristics of supply chains, this paper explores the class Hotelling model for describing sequential auctions with some particular assumptions under second-price sealed-bid auction mechanisms. In the proposed model of the current study, both close substitutes and bidders are hypothetically distributed at the interval [0,1]; a bidder type is continuous and multi-dimensional (namely, each bidder has different valuations for each close substitute); each bidder's valuations for close substitutes are not independent. Moreover, this model can skillfully convert multi-dimensional types into one-dimensional types via interdependence of distances between bidders and items in $[0,1]$.
The rest of this paper is organized as follows: Section 2 states formally the sequential auctions of close substitutes with associated valuations and constructs a class Hotelling model. Section 3 proves the existence of equilibrium bids based on the model under the second-price sealed-bid auction format and explores some conditions of efficient auctions. Further, information policies, equilibrium bids, and overall revenues of the sequential auctions of close substitutes with especially associated valuations are specifically deduced and discussed in Section 4. Relevant conclusions are summarized in Section 5.

\section{A class Hotelling model for describing sequential auctions}

It is supposed that two close substitutes, Items A and $\mathrm{B}$, are auctioned sequentially for $n \geq 3$ bidders via second-price sealed-bid auctions. Auction rules and some assumptions are as follows:

1. Item A is sold at the first auction, and Item B is sold at the second one. In addition, at each auction, the bidder with the highest bid wins and pays the second highest bid. A tie is broken by rolling a fair coin;

2. Each bidder is risk neutral and has a unit-demand; accordingly, the bidder with Item $A$ will exit the second auction;

3. Both items and bidders are distributed at the interval $[0,1]$ and mutually stochastically independent, as Figure 1 shows. The location of each bidder is his or her private information;

4. Each bidder's valuation for Item $k \in\{\mathrm{A}, \mathrm{B}\}$ is $V_{k}-$ $\tau\left(d_{k}\right)$, where $\tau\left(d_{k}\right) \geq 0, \tau^{\prime}\left(d_{k}\right)>0$, and $d_{k}$ denotes the distance between the bidder and Item $k$. Here, the value of Item $k \in\{\mathrm{A}, \mathrm{B}\}, V_{k} \geq 1$ is commonly known.

The last assumption is inspired by the standard Hotelling model [15]. When making trades in real-life markets, it is imperative to consider the factor of cost in general and its various types such as transportation costs or maintenance costs, which usually increase the functions of transportation distances. In this paper, $V_{k}$ is the same for all bidders. However, when taking into account transportation cost $\tau\left(d_{k}\right)$, each bidder's true valuation for $k \in\{\mathrm{A}, \mathrm{B}\}$ should be $V_{k}-\tau\left(d_{k}\right)$, which is different from those of other bidders.

Figure 1 shows the class Hotelling model. In Figure 1, the locations of Items A and B are denoted by $\alpha \in[0,1]$ and $\beta \in[0,1]$, respectively. Without loss

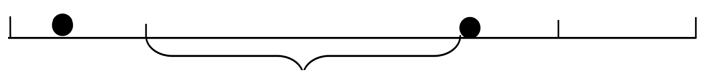

Figure 1. The class Hotelling model. 
of generality, it is assumed that $\alpha \leq \beta$. The location of a bidder at the interval is denoted by $t \in[0,1]$. To simplify the expressions, Bidder $t$ is used to denote the bidder whose location in $[0,1]$ is $t$. Thus, the distance between Bidder $t$ and Item $\mathrm{A}$ is $d_{\mathrm{A}}(t)=|\alpha-t|$, and one between Bidder $t$ and Item $\mathrm{B}$ is $d_{\mathrm{B}}(t)=|\beta-t|$. Accordingly, $v_{k}(t)=V_{k}-\tau\left(d_{k}(t)\right)(k \in\{\mathrm{A}, \mathrm{B}\})$ is Bidder $t$ 's valuation for Item $k \in\{\mathrm{A}, \mathrm{B}\}$. Furthermore, let $b_{k}(t)$ represent Bidder $t$ 's bid for $k \in\{\mathrm{A}, \mathrm{B}\}$.

Remark 1. In Figure 1, items and bidders correspond to firms and customers in the standard Hotelling model, respectively. While $V_{\mathrm{A}}=V_{\mathrm{B}}, \beta-\alpha=0$ means that the close substitutes (Items A and B) are identical for bidders, and $\beta-\alpha=1$ means that maximum differences exist among them. Moreover, similar to the preferences in the standard Hotelling model, the smaller the distance $d_{k}(t)(k \in\{\mathrm{A}, \mathrm{B}\})$ is, the greater Bidder $t$ 's valuation for item $k$ will be.

Remark 2. In Figure 1, bidders' valuations for two items are their own private information and independent among bidders; however, each bidder's private valuations for two items $v_{\mathrm{A}}(t)=V_{\mathrm{A}}-\tau\left(d_{\mathrm{A}}(t)\right)$ and $v_{\mathrm{B}}(t)=V_{\mathrm{B}}-\tau\left(d_{\mathrm{B}}(t)\right)$ are correlated because either $\left|d_{\mathrm{A}}(t)-d_{\mathrm{B}}(t)\right|=\beta-\alpha$ or $d_{\mathrm{A}}(t)+d_{\mathrm{B}}(t)=\beta-\alpha$, while $\alpha$ and $\beta$ are given in advance. Thus, the model in Figure 1 can be used approximatively to describe sequential auctions of close substitutes with particular assumptions of non-independent valuations, under which the probability of Bidder $t$ winning Item $B$ is intuitively relevant to one of his or her winning A. Concurrently, by means of $t$, our model skillfully converts a two-dimensional type of a bidder into a onedimensional one. Thus, $t$ may be also regarded as the bidder type.

\section{Equilibrium and winners}

Because the second stage of the sequential auctions in this paper is actually a private-value second-price sealed-bid auction, Bidder t's dominant strategy for Item $\mathrm{B}$ is to bid his or her own valuation for Item B, namely $b_{\mathrm{B}}(t)=v_{\mathrm{B}}(t)$. However, deciding the bid for Item $\mathrm{A}$ is more complicated than that for Item $\mathrm{B}$, because Bidder $t$ will consider his or her expected surplus in the second stage.

Let:

$$
x=\max _{\theta \neq t} b_{\mathrm{A}}(\theta),
$$

be the highest bid of Bidder $t$ 's $n-1$ opponents in the first stage. If $b_{\mathrm{A}}(t)>x$, then Bidder $t$ wins at the first auction and his or her surplus is $v_{\mathrm{A}}(t)-x$. If $b_{\mathrm{A}}(t)<x$, then Bidder $t$ loses at the first auction and all his or her remaining opponents at the second auction belong to $\Omega(x)=\left\{t^{\prime} \mid b_{\mathrm{A}}\left(t^{\prime}\right) \leq x\right\}$, which is a set of bidders whose bids for Item A are not more than $x$. Further, let:

$$
\Phi_{n-2 /-t}(z, \Omega(x))=P\left(\max _{\theta \in \Omega(x) \text { and } \theta \neq t} v_{\mathrm{B}}(\theta) \leq z\right),
$$

be the probability distribution function of the highest valuation of the remaining bidders (except $t$ ) at the second auction. Then, Bidder $t \in \Omega(x)$ can win Item $\mathrm{B}$ with a probability of $\Phi_{n-2 /-t}\left(v_{\mathrm{B}}(t), \Omega(x)\right)$, and his expected surplus at the second auction is as follows:

$$
\sigma\left(v_{\mathrm{B}}(t), x\right)=\int_{0}^{v_{\mathrm{B}}(t)}\left(v_{\mathrm{B}}(t)-z\right) d \Phi_{n-2 /-t}(z, \Omega(x)) .
$$

Obviously, winning Item $\mathrm{A}$ is more beneficial to $t$ if and only if $v_{\mathrm{A}}(t)-x>\sigma\left(v_{\mathrm{B}}(t), x\right)$. In addition, a logical and ideal bid $b_{\mathrm{A}}(t)$ should satisfy $v_{\mathrm{A}}(t)-b_{\mathrm{A}}(t)=$ $\sigma\left(v_{\mathrm{B}}(t), b_{\mathrm{A}}(t)\right)$ so that it would not make any difference for Bidder $t$ to win Item $\mathrm{A}$ or to win Item B. In addition, because $v_{\mathrm{A}}(t) \leq \sigma\left(v_{\mathrm{B}}(t), 0\right)$ implies that giving up Item $\mathrm{A}$ is always a good choice for Bidder $t$ even though all other bidders bid 0 or do not submit their bids at the first auction, it is assumed here that $v_{\mathrm{A}}(t)>\sigma\left(v_{\mathrm{B}}(t), 0\right)$ holds for $\forall t \in[0,1]$ in this paper. Inspired by Zeithammer [14], Proposition 1 gives necessary and sufficient conditions of equilibrium bid $b_{\mathrm{A}}(t)$.

Proposition 1. Suppose that $v_{\mathrm{A}}(t)>\sigma\left(v_{\mathrm{B}}(t), 0\right)$ for $\forall t \in[0,1)$. If and only if $1+\frac{\partial \sigma\left(v_{\mathrm{B}}(t), x\right)}{\partial x}>0$ for $\forall t \in[0,1]$, there is a unique equilibrium bid $b_{\mathrm{A}}(t)$ that satisfies the following equation:

$$
b_{\mathrm{A}}(t)=v_{\mathrm{A}}(t)-\sigma\left(v_{\mathrm{B}}(t), b_{\mathrm{A}}(t)\right) .
$$

Proof. Let $S(x)=v_{\mathrm{A}}(t)-x-\sigma\left(v_{\mathrm{B}}(t), x\right)$. Proposition 1 needs to be proved based on the following two aspects:

1. $S(x)=0$ has a unique solution, $x_{0}>0$, if and only if $1+\frac{\partial \sigma\left(v_{\mathrm{B}}(t), x\right)}{\partial x}>0$ for $\forall t \in[0,1]$;

2. $x_{0}>0$ is Bidder $t$ 's equilibrium bid for Item A.

The solution under this condition can be divided into the following two cases to discuss:

i. If $1+\frac{\partial \sigma\left(v_{\mathrm{B}}(t), x\right)}{\partial x}>0$, then $S^{\prime}(x)=-1-\frac{\partial \sigma\left(v_{\mathrm{B}}(t), x\right)}{\partial x}<$ 0 . Therefore, $S(x)$ strictly monotonically decreases with $x$. According to $v_{\mathrm{A}}(t)>\sigma\left(v_{\mathrm{B}}(t), 0\right)$ for $\forall t \in$ $[0,1)$, we have $S(0)>0$. Moreover, $S\left(v_{\mathrm{A}}(t)\right)=$ $v_{\mathrm{A}}(t)-v_{\mathrm{A}}(t)-\sigma\left(v_{\mathrm{B}}(t), v_{\mathrm{A}}(t)\right) \leq 0$. Because $S(x)$ is continuous and strictly monotonic with $\mathrm{x}$, there must exist a unique solution $x_{0} \in\left(0, v_{\mathrm{A}}(t)\right]$ satisfying $S\left(x_{0}\right)=0$.

Suppose that $1+\frac{\partial \sigma\left(v_{\mathrm{B}}(t), x\right)}{\partial x} \leq 0$, then $S^{\prime}(x)=$ $-1-\frac{\partial \sigma\left(v_{\mathrm{B}}(t), x\right)}{\partial x} \geq 0$. For instance, $S(x)$ monotonically increases with $x$. Thus, for $\forall x>0$, 
$S(x) \geq S(0)=v_{\mathrm{A}}(t)-\sigma\left(v_{\mathrm{B}}(t), 0\right)>0$, which conflicts with the condition that $S(x)=0$ has one positive solution: $x_{0}>0$. Thus, $1+\frac{\partial \sigma\left(v_{\mathrm{B}}(t), x\right)}{\partial x}>$ 0 ;

ii. It is assumed that $b_{\mathrm{A}}(t)<x_{0}$. While $b_{\mathrm{A}}(t)<$ $x_{0}<x=\max _{\theta \neq t}\left(b_{\mathrm{A}}(\theta)\right)$ or $x<b_{\mathrm{A}}(t)<x_{0}$, Bidder $t$ is indifferent to bid $x_{0}$ or $b_{\mathrm{A}}(t)$. While $b_{\mathrm{A}}(t)<x<x_{0}$, Bidder $t$ will lose Item $\mathrm{A}$ and his expected surplus at the second auction is $\sigma\left(v_{\mathrm{B}}(t), x\right)$. Notice that $S(x)>S\left(x_{0}\right)=0$ because $S(x)$ strictly monotonically decreases with $x<x_{0}$. Accordingly, $\sigma\left(v_{\mathrm{B}}(t), x\right)<v_{\mathrm{A}}(t)-x$. Notice that $v_{\mathrm{A}}(t)-x$ is Bidder $t$ 's surplus if he or she bids $x_{0}$ and wins Item $\mathrm{A}$. Therefore, bidding $b_{\mathrm{A}}(t)<x_{0}$ is worse than bidding $x_{0}$ for Bidder $t$.

A similar proof shows that bidding $b_{\mathrm{A}}(t)>x_{0}$ is worse for Bidder $t$. Therefore, $b_{\mathrm{A}}(t)=x_{0}$ is Bidder $t$ 's equilibrium bid for Item $\mathrm{A}$.

Remark 3. Essentially, $1+\frac{\partial \sigma\left(v_{\mathrm{B}}(t), x\right)}{\partial x}>0$ is the sufficient and necessary condition of $v_{\mathrm{A}}(t)-\sigma\left(v_{\mathrm{B}}(t), x\right)$ that has a fixed point, which is Bidder $t$ 's equilibrium bid for Item A. Here, $v_{\mathrm{A}}(t)-b_{\mathrm{A}}(t)$ and $\sigma\left(v_{\mathrm{B}}(t), b_{\mathrm{A}}(t)\right)$ are Bidder $t$ 's expected surplus at the first auction and that at the second auction, respectively. Accordingly, Proposition 1 implies that winning Item A or B should yield the same expected surplus for Bidder $t$ while he or she determines the optimal bid for Item A. Intuitively, Proposition 1 is also appropriate for sequential auctions of more than two items while Item A is regarded as the current item and Item B as the sum of all follow-up (or future) items.

Based on Proposition 1, Propositions 2 and 3 identify the winner at the first auction.

Proposition 2. If $\tau\left(d_{k}\right)(k \in\{\mathrm{A}, \mathrm{B}\})$ is a linear function of $d_{k}, \tau^{\prime}\left(d_{k}\right)>0$, and $1+\frac{\partial \sigma\left(v_{\mathrm{B}}(t), x\right)}{\partial x}>0$, then Bidder $t=\arg \min _{t_{1}, t_{2}, \cdots, t_{n}} d_{\mathrm{A}}\left(t_{i}\right)=\arg \min _{t_{1}, t_{2}, \cdots, t_{n}}\left|t_{i}-\alpha\right|$ will win Item A.

Proof. Proposition 1 indicates that if $1+\frac{\partial \sigma\left(v_{\mathrm{B}}(t), x\right)}{\partial x}>$ 0 , then equilibrium bid $b_{\mathrm{A}}(t)$ for Item $\mathrm{A}$ is an implicit function satisfying Eq. (2). According to $v_{\mathrm{A}}(t)=V_{\mathrm{A}}-$ $\tau\left(d_{\mathrm{A}}(t)\right)$ and $v_{\mathrm{B}}(t)=V_{\mathrm{B}}-\tau\left(d_{\mathrm{B}}(t)\right)$, we have:

$$
\begin{aligned}
\frac{d b_{\mathrm{A}}(t)}{d d_{\mathrm{A}}(t)}= & \frac{d v_{\mathrm{A}}(t)}{d d_{\mathrm{A}}(t)}-\frac{\partial \sigma\left(v_{\mathrm{B}}(t), b_{\mathrm{A}}(t)\right)}{\partial v_{\mathrm{B}}(t)} \cdot \frac{d v_{\mathrm{B}}(t)}{d d_{\mathrm{A}}(t)} \\
& -\frac{\partial \sigma\left(v_{\mathrm{B}}(t), b_{\mathrm{A}}(t)\right)}{\partial b_{\mathrm{A}}(t)} \cdot \frac{d b_{\mathrm{A}}(t)}{d d_{\mathrm{A}}(t)} .
\end{aligned}
$$

Namely:

$$
\begin{aligned}
\frac{d b_{\mathrm{A}}(t)}{d d_{\mathrm{A}}(t)}= & {\left[-\frac{d \tau\left(d_{\mathrm{A}}(t)\right)}{d d_{\mathrm{A}}(t)}+\frac{\partial \sigma\left(v_{\mathrm{B}}(t), b_{\mathrm{A}}(t)\right)}{\partial v_{\mathrm{B}}(t)}\right.} \\
& \left.\cdot \frac{d \tau\left(d_{\mathrm{B}}(t)\right)}{d d_{\mathrm{B}}(t)} \cdot \frac{d d_{\mathrm{B}}(t)}{d d_{\mathrm{A}}(t)}\right] \\
& {\left[1+\frac{\partial \sigma\left(v_{\mathrm{B}}(t), b_{\mathrm{A}}(t)\right)}{\partial b_{\mathrm{A}}(t)}\right]^{-1} } \\
= & w\left[-1+\frac{\partial \sigma\left(v_{\mathrm{B}}(t), b_{\mathrm{A}}(t)\right)}{\partial v_{\mathrm{B}}(t)} \cdot \frac{d d_{\mathrm{B}}(t)}{d d_{\mathrm{A}}(t)}\right] \\
& {\left[1+\frac{\partial \sigma\left(v_{\mathrm{B}}(t), b_{\mathrm{A}}(t)\right)}{\partial b_{\mathrm{A}}(t)}\right]^{-1} \cdot }
\end{aligned}
$$

In Eq. (3), $w=\frac{d \tau\left(d_{\mathrm{A}}(t)\right)}{d d_{\mathrm{A}}(t)}=\frac{d \tau\left(d_{\mathrm{B}}(t)\right)}{d d_{\mathrm{B}}(t)}>0$ because $\tau\left(d_{k}\right)(k \in\{\mathrm{A}, \mathrm{B}\})$ is a linear function of $d_{k}$ and $\tau^{\prime}\left(d_{k}\right)>0$. Moreover, obviously:

$$
\frac{\partial \sigma\left(v_{\mathrm{B}}(t), b_{\mathrm{A}}(t)\right)}{\partial v_{\mathrm{B}}(t)}=\Phi_{n-2 /-t}\left(v_{\mathrm{B}}(t), \Omega\left(b_{\mathrm{A}}(t)\right)\right) \leq 1,
$$

and:

$$
1+\frac{\partial \sigma\left(v_{\mathrm{B}}(t), b_{\mathrm{A}}(t)\right)}{\partial b_{\mathrm{A}}(t)}>0 .
$$

If $\alpha<\beta \leq t$, then $d_{\mathrm{B}}(t)=t-\beta=(t-\alpha)-$ $(\beta-\alpha)=d_{\mathrm{A}}(t)-(\beta-\alpha)$; if $\alpha \leq t<\beta$, then $d_{\mathrm{B}}(t)=\beta-t=(\beta-\alpha)-(t-\alpha)=(\beta-\alpha)-d_{\mathrm{A}}(t)$; if $t<\alpha<\beta$, then $d_{\mathrm{B}}(t)=\beta-t=(\beta-\alpha)+$ $(\alpha-t)=(\beta-\alpha)+d_{\mathrm{A}}(t)$. Accordingly, $\left|\frac{d d_{\mathrm{B}}(t)}{d d_{\mathrm{A}}(t)}\right|=1$. Thus, $\frac{d b_{\mathrm{A}}(t)}{d d_{\mathrm{A}}(t)} \leq 0$, that is, $b_{\mathrm{A}}(t)$ is a decreasing function of $d_{\mathrm{A}}(t)$ and an increasing function of $v_{\mathrm{A}}(t)=V_{\mathrm{A}}-$ $\tau\left(d_{\mathrm{A}}(t)\right)$. Therefore, Bidder $t$ nearest to Item A (i.e., $\left.t=\arg \min _{t_{1}, t_{2}, \cdots t_{n}} d_{\mathrm{A}}\left(t_{i}\right)=\arg \min _{t_{1}, t_{2}, \cdots t_{n}}\left|t_{i}-\alpha\right|\right)$ will win Item $\mathrm{A}$ at the first auction.

As a direct result of Proposition 2, the following corollary is obtained.

Corollary 1. The auction for Item A is efficient, while $\tau\left(d_{k}\right)(k \in\{\mathrm{A}, \mathrm{B}\})$ is linear, $\tau^{\prime}\left(d_{k}\right)>0$, and $1+\frac{\partial \sigma\left(v_{\mathrm{B}}(t), b\right)}{\partial b}>0$.

While $\tau\left(d_{k}\right)(k \in\{\mathrm{A}, \mathrm{B}\})$ is a non-linear function of $d_{k}$, which is contingent on predicting the winner at the first auction in advance. For example, let $\tau\left(d_{k}\right)=$ $1-e^{-d_{k}}$ and $\forall t \geq \beta>\alpha=0$, namely all bidders are on the right-hand side of Item B in Figure 1. Then:

$$
d_{\mathrm{A}}(t)=t, \quad d_{\mathrm{B}}(t)=t-\beta,
$$

and:

$$
\frac{d b_{\mathrm{A}}(t)}{d d_{\mathrm{A}}}(t)=\frac{d b_{\mathrm{A}}(t)}{d t}
$$




$$
\begin{aligned}
& =e^{-t}\left[-1+e^{\beta} \Phi_{n-2 /-t}\left(v_{\mathrm{B}}(t), \Omega\left(b_{\mathrm{A}}(t)\right)\right)\right] \\
& {\left[1+\frac{\partial \sigma\left(v_{\mathrm{B}}(t), b_{\mathrm{A}}(t)\right)}{\partial b_{\mathrm{A}}(t)}\right]^{-1} .}
\end{aligned}
$$

If $\beta$ is large enough, then $\frac{d b_{\mathrm{A}}(t)}{d d_{\mathrm{A}}(t)}$ or $\frac{d b_{\mathrm{A}}(t)}{d t}$ may be more than zero, which means that $b_{\mathrm{A}}(t)$ may be an increasing function of $d_{\mathrm{A}}(t)$ or $t$. However, $v_{\mathrm{A}}(t)=$ $V_{\mathrm{A}}-\tau\left(d_{\mathrm{A}}(t)\right)=V_{\mathrm{A}}-1+e^{-d_{\mathrm{A}}(t)}=V_{\mathrm{A}}-1+e^{-t}$ is a decreasing function of $d_{\mathrm{A}}(t)$ or $t$. Therefore, the auction for Item A may be inefficient.

Proposition 3. If $\tau^{\prime}\left(d_{k}\right) \geq 0, \tau^{\prime \prime}\left(d_{k}\right) \geq 0, k \in$ $\{\mathrm{A}, \mathrm{B}\}, 1+\frac{\partial \sigma\left(v_{\mathrm{B}}(t), x\right)}{\partial x}>0$, and $t_{i} \geq \alpha(i=1,2, \cdots, n)$, then Bidder $t=\arg \min _{t_{1}, t_{2}, \cdots t_{n}} d_{\mathrm{A}}\left(t_{i}\right)=\arg \min _{t_{1}, t_{2}, \cdots t_{n}} \mid t_{i}-$ $\alpha \mid$ will win Item $\mathrm{A}$.

Proof. Notice that $t_{i} \geq \alpha(i=1,2, \cdots, n)$ means that all bidders on the right-hand side of Item $A$ in $[0,1]$. According to Proposition 1, Bidder $t$ 's equilibrium bid is $b_{\mathrm{A}}(t)=v_{\mathrm{A}}(t)-\sigma\left(v_{\mathrm{B}}(t), b_{\mathrm{A}}(t)\right)$. Because $v_{\mathrm{A}}(t)=V_{\mathrm{A}}-\tau\left(d_{\mathrm{A}}(t)\right)$ and $v_{\mathrm{B}}(t)=V_{\mathrm{B}}-\tau\left(d_{\mathrm{B}}(t)\right)$, we have:

$$
\begin{gathered}
\frac{d b_{\mathrm{A}}(t)}{d d_{\mathrm{A}}(t)}=\left[-\frac{d \tau\left(d_{\mathrm{A}}(t)\right)}{d d_{\mathrm{A}}(t)}+\frac{\partial \sigma\left(v_{\mathrm{B}}(t), b_{\mathrm{A}}(t)\right)}{\partial v_{\mathrm{B}}(t)}\right. \\
\left.\cdot \frac{d \tau\left(d_{\mathrm{B}}(t)\right)}{d d_{\mathrm{B}}(t)} \cdot \frac{d d_{\mathrm{B}}(t)}{d d_{\mathrm{A}}(t)}\right]\left[1+\frac{\partial \sigma\left(v_{\mathrm{B}}(t), b_{\mathrm{A}}(t)\right)}{\partial b_{\mathrm{A}}(t)}\right]^{-1}
\end{gathered}
$$

Notice that:

$$
\frac{\partial \sigma\left(v_{\mathrm{B}}(t), b_{\mathrm{A}}(t)\right)}{\partial v_{\mathrm{B}}(t)}=\Phi_{n-2 /-t}\left(v_{\mathrm{B}}(t), \Omega\left(b_{\mathrm{A}}(t)\right)\right) \leq 1,
$$

and:

$$
1+\frac{\partial \sigma\left(v_{\mathrm{B}}(t), b_{\mathrm{A}}(t)\right)}{\partial b_{\mathrm{A}}(t)}>0
$$

If $t \geq \beta$, then $d_{\mathrm{B}}(t)=t-\beta=d_{\mathrm{A}}(t)-(\beta-\alpha) \leq d_{\mathrm{A}}(t)$ $(\because t \geq \alpha)$ and $\beta \geq \alpha$. Accordingly, $\frac{d d_{\mathrm{B}}(t)}{d d_{\mathrm{A}}(t)}=1$ and $\frac{d \tau\left(d_{\mathrm{A}}(t)\right)}{d d_{\mathrm{A}}(t)} \geq \frac{d \tau\left(d_{\mathrm{B}}(t)\right)}{d d_{\mathrm{B}}(t)} \geq 0\left(\because \tau^{\prime}\left(d_{k}\right) \geq 0, \tau^{\prime \prime}\left(d_{k}\right) \geq 0\right.$ and $k \in\{\mathrm{A}, \mathrm{B}\})$. Hence, $\frac{d b_{\mathrm{A}}(t)}{d d_{\mathrm{A}}(t)} \leq 0$.

If $t<\beta$, then $d_{\mathrm{B}}(t)=\beta-t=(\beta-\alpha)-d_{\mathrm{A}}(t) \geq 0$ $(\because t \geq \alpha=0$ and $\beta \geq \alpha)$. Accordingly, $\frac{d d_{\mathrm{B}}(t)}{d d_{\mathrm{A}}(t)}=-1$. In addition $\frac{d \tau\left(d_{\mathrm{B}}(t)\right)}{d d_{\mathrm{B}}(t)} \geq 0$ and $\frac{d \tau\left(d_{\mathrm{A}}(t)\right)}{d d_{\mathrm{A}}(t)} \geq 0\left(\because \tau^{\prime}\left(d_{k}\right) \geq 0\right.$, $k \in\{\mathrm{A}, \mathrm{B}\})$. Hence, $\frac{d b_{\mathrm{A}}(t)}{d d_{\mathrm{A}}(t)} \leq 0$.

As a result, $b_{\mathrm{A}}(t)$ is a decreasing function of $d_{\mathrm{A}}(t)$ or $t$. Therefore, Bidder $t$ nearest to Item A (i.e., $t=$ $\left.\arg \min _{t_{1}, t_{2}, \cdots, t_{n}} d_{\mathrm{A}}\left(t_{i}\right)=\arg \min _{t_{1}, t_{2}, \cdots, t_{n}}\left|t_{i}-\alpha\right|\right)$ will submit the highest bid and win Item $\mathrm{A}$.

Proposition 3 actually gives nonlinear conditions of efficient auctions in the class Hotelling model. Concurrently, its proof implies the following corollary.
Corollary 2. Let $\alpha \leq \beta, \tau^{\prime}\left(d_{k}\right) \geq 0, k \in\{\mathrm{A}, \mathrm{B}\}$, and $1+\frac{\partial \sigma\left(v_{\mathrm{B}}(t), x\right)}{\partial x}>0$. The auction for Item $\mathrm{A}$ is efficient while any one of the following conditions is satisfied:

1. $\tau^{\prime \prime}\left(d_{k}\right) \geq 0$ and $t_{i} \geq \alpha(i=1,2, \cdots, n)$; all bidders are on the right-hand side of Item $\mathrm{A}$;

2. $\tau^{\prime \prime}\left(d_{k}\right)<0$ and $t_{i} \leq \beta(i=1,2, \cdots, n)$; all bidders are on the left-hand side of Item B;

3. All bidders are between A and B;

4. $\tau\left(d_{k}\right)$ is linear, and Bidders' locations are arbitrary in $[0,1]$.

Remark 4. Corollary 2 shows that the efficiency of the sequential auctions described by the class Hotelling model is conditional. More specifically, locations of suppliers and agents in a traffic line should be considered for the auctions of supply contracts in Section 1.

\section{Bids and information policies about $\boldsymbol{\beta}$}

The key to determining the equilibrium bid $b_{\mathrm{A}}(t)=$ $v_{\mathrm{A}}(t)-\sigma\left(v_{\mathrm{B}}(t), b_{\mathrm{A}}(t)\right)$ in Proposition 1 is the expected surplus:

$$
\begin{gathered}
\sigma\left(v_{\mathrm{B}}(t), b_{\mathrm{A}}(t)\right)=\int_{0}^{v_{\mathrm{B}}(t)}\left(v_{\mathrm{B}}(t)-z\right) \\
d \Phi_{n-2 /-t}\left(z, \Omega\left(b_{\mathrm{A}}(t)\right)\right),
\end{gathered}
$$

in which both $v_{\mathrm{B}}(t)$ and $\Phi_{n-2 /-t}\left(z, \Omega\left(b_{\mathrm{A}}(t)\right)\right)$ are closely related to the location of Item B. Thus, in order to obtain a concrete formula of $\sigma\left(v_{\mathrm{B}}(t), b_{\mathrm{A}}(t)\right)$, we need to consider information policies about $\beta \in[0,1]$, namely revealing Item $\mathrm{B}$ or hiding Item $\mathrm{B}$ before the auction of Item A ends. Here, revealing Item B means that all bidders know $\beta$, and hiding Item $\mathrm{B}$ means that all bidders do not know $\beta$ until the first auction ends. In this section, we will apply the class Hotelling model to deduce some concrete equilibrium bids of sequential auctions of close substitutes.

Intuitively, any bidder at auctions subjectively regards himself or herself as a marginal loser [12] or winner, because he or she will rationally give up auctions if he or she finds no opportunities to win. Here, a marginal loser or winner respectively points to the loser with the highest bid or the winner with the lowest bid. Specifically, in our class Hotellling model with $\tau\left(d_{k}\right)=d_{k}$, the bidder nearest to Item A will win according to Proposition 2 or Proposition 3. Thus, Bidder $t$ regarding himself or herself as a marginal loser or winner of Item A always believes that the rest of his or her opponents are distributed on his right side (namely in $[t, 1]$ ), except none of his opponents distributed in $[0, t)$. Such an idea of a preponderant rival exactly leads to the bidding equilibrium mentioned by Proposition 1. For convenience, Bidder $t$ is called a 
preponderant rival of Item $\mathrm{A}$ if and only if there is at most one opponent in $[0, t)$.

In order to facilitate discussions, it is assumed in this section that:

1. $V_{\mathrm{A}}=1$ and $V_{\mathrm{B}}=1$;

2. $\alpha=0$ and $\tau\left(d_{k}\right)=d_{k}$ for $k \in\{\mathrm{A}, \mathrm{B}\}$.

The above assumptions imply that all bidders are on the right-hand side of Item $\mathrm{A}$, namely $t \geq \alpha=0$, and $\tau\left(d_{k}\right)$ is linear. Furthermore, let $f(x)(F(x))$ and $g(x)(G(x))$ denote the probability density (probability distribution) of $t$ and one of $\beta$, respectively.

By means of Proposition 1 and Corollary 2, we will deduce the expected surplus $\sigma\left(v_{\mathrm{B}}(t), b_{\mathrm{A}}(t)\right)$ of Bidder $t$ as a preponderant rival and verify and prove that $b_{\mathrm{A}}(t)=v_{\mathrm{A}}(t)-\sigma\left(v_{\mathrm{B}}(t), b_{\mathrm{A}}(t)\right)$ is exactly his or her equilibrium bid for Item $\mathrm{A}$.

\subsection{Equilibrium bids when revealing $\beta$}

Each bidder knows Item A' location $\alpha=0$, Item B's location $\beta \in[0,1]$, his or her own location (or type) $t \in[0,1]$, and bidders' number $n$ and does not know the locations (or types) of other bidders. However, he or she regards other bidders' locations as random variables distributed in $[0,1]$. First, all $n$ bidders submit their sealed bids for Item A. Then, the bidder with the highest bid wins Item A, pays the second highest bid, and exits the second auction. Next, the remaining $n-1$ bidders submit their sealed bids for Item B. Finally, the bidder with the highest bid wins Item $\mathrm{B}$ and pays the second highest bid.

First, let $\pi_{x}^{y}\left(v_{\mathrm{B}}(t)\right)$ denote Bidder $t$ 's expected surplus of winning Item B while he or she faces a group of opponents distributed at the interval $[x, y]$, where $x, y \in[0,1]$ and $x \leq y$. According to Proposition 2, a preponderant rival of Item A can only defeat the bidders who are far away from Item A. Obviously, the probability density of the location of an opponent in $[t, 1]$ is $\frac{f(s)}{1-F(t)}$, in which $s \in[t, 1]$. Thus, the probability and the probability density that all locations of $n-2$ opponents in $[t, 1]$ are greater than $s \in[t, 1]$ should be $\left(\frac{1-F(s)}{1-F(t)}\right)^{n-2}$ and $\frac{n-2}{1-F(t)}\left(\frac{1-F(s)}{1-F(t)}\right)^{n-3}$, respectively. Therefore, while Bidder $t$ only considers opponents on his or her right side, his or her expected surplus of winning Item B should be:

$$
\begin{gathered}
\pi_{x}^{y}\left(v_{\mathrm{B}}(t)\right)=\int_{x}^{y} \frac{n-2}{1-F(t)}\left(\frac{1-F(s)}{1-F(t)}\right)^{n-3} \\
\left(v_{\mathrm{B}}(t)-v_{\mathrm{B}}(s)\right) d s
\end{gathered}
$$

where $t \leq x \leq y \leq 1$.

Then, in order to determine the expected surplus $\sigma\left(v_{\mathrm{B}}(t), b_{\mathrm{A}}(t)\right)$ of Bidder $t$ as a preponderant rival of Item $\mathrm{A}$ in Figure 2, the interval $[0,1]$ is divided into

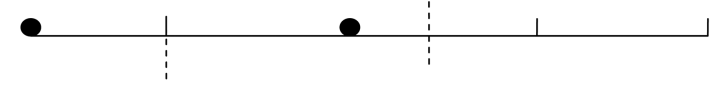

Figure 2. The case of $W_{2}$.

three subintervals, namely $W 1=[0, t], W 2=[t,(1+$ $t) / 2]$, and $W 3=[(1+t) / 2,1]$. Thus, three cases need to be considered for $\beta$.

Figure 2 shows the case of $W 2$. By observing Figure 2, the optimal solution $t^{*}$ of Eq. (2) denotes the symmetric of $t$ in $[0,1]$ with respect to $\beta$, namely $t^{*}=2 \beta-t$. Obviously, bids of opponents in $\left[t, t^{*}\right]$ for Item $\mathrm{B}$ should be greater than one of Bidder $t$. Again, because opponents are distributed in $[0,1], \sigma\left(v_{\mathrm{B}}(t), b_{\mathrm{A}}(t)\right)$ is only relevant to the opponents in $[0, t]$ and $\left[t^{*}, 1\right]$. As a preponderant rival of Item $\mathrm{A}$, Bidder $t$ subjectively believes that his or her $n-2$ opponents in the second stage should be distributed on his or her right side, namely in $[t, 1]$. Therefore, $\sigma\left(v_{\mathrm{B}}(t), b_{\mathrm{A}}(t)\right)=$ $\pi_{t^{*}}^{1}\left(v_{\mathrm{B}}(t)\right)$. In addition, the other cases can be inferred similarly.

Finally, a concrete formula of $\sigma\left(v_{\mathrm{B}}(t), b_{\mathrm{A}}(t)\right)$ is related to the specific probability distribution of $t$ and one of $\beta$. Further, it is assumed in Section 4 that $t$ and $\beta$ are uniformly distributed in $[0,1]$.

To be specific, $f(x)=1, g(x)=1, F(x)=x$, and $G(x)=x$ while $x \in[0,1]$. In conclusion, the expected surplus $\sigma\left(v_{\mathrm{B}}(t), b_{\mathrm{A}}(t)\right)$ in various cases is shown below.

1) $\beta \in W 1$ :

$$
\begin{aligned}
& \sigma\left(v_{\mathrm{B}}(t), b_{\mathrm{A}}(t)\right)=\pi_{t}^{1}\left(v_{\mathrm{B}}(t)\right) \\
& =\int_{t}^{1} \frac{n-2}{1-F(t)}\left(\frac{1-F(s)}{1-F(t)}\right)^{n-3}(s-t) d s=\frac{1-t}{n-1} .
\end{aligned}
$$

2) $\beta \in W 2$ :

$$
\begin{aligned}
\sigma & \left(v_{\mathrm{B}}(t), b_{\mathrm{A}}(t)\right)=\pi_{2 \beta-t}^{1}\left(v_{\mathrm{B}}(t)\right) \\
& =\int_{2 \beta-t}^{1} \frac{n-2}{1-F(t)}\left(\frac{1-F(s)}{1-F(t)}\right)^{n-3}(t+s-2 \beta) d s \\
& =\frac{(1-2 \beta+t)^{n-1}}{(n-1)(1-t)^{n-2}} .
\end{aligned}
$$

3) $\beta \in W 3$ :

$$
\sigma\left(v_{\mathrm{B}}(t), b_{\mathrm{A}}(t)\right)=0 .
$$

Proposition 4. If $\beta$ is revealed before auctioning Item $\mathrm{A}$, then Bidder $t$ 's equilibrium bid for Item $\mathrm{A}$ is $b_{\mathrm{A}}(t)=1-t-\sigma\left(v_{\mathrm{B}}(t), b_{\mathrm{A}}(t)\right)$, where $\sigma\left(v_{\mathrm{B}}(t), b_{\mathrm{A}}(t)\right)$ is shown as in Eqs. (5), (6), and (7). 
Proof. Let $x=b_{\mathrm{A}}\left(t_{x}\right)=\max _{\theta \neq t} b_{\mathrm{A}}(\theta)$ be the highest bid of Bidder $t$ 's $n-1$ opponents in the first stage and $h(x)$ denote the probability density of $x$. Usually, equilibrium bid $b_{\mathrm{A}}(t)$ should maximize Bidder $t$ 's expected surplus $\tau\left(b_{\mathrm{A}}(t)\right)$ at the first auction, namely:

$$
\begin{aligned}
\max _{b_{\mathrm{A}}(t)} \tau\left(b_{\mathrm{A}}(t)\right)= & \max _{b_{\mathrm{A}}(t)}\left(\int_{0}^{b_{\mathrm{A}}(t)}\left(V_{\mathrm{A}}(t)-x\right) h(x) d x\right. \\
& \left.+\int_{b_{\mathrm{A}}(t)}^{1} \sigma\left(V_{\mathrm{B}}(t), x\right) h(x) d x\right) .
\end{aligned}
$$

Let:

$$
\begin{aligned}
\frac{d \tau\left(b_{\mathrm{A}}(t)\right)}{d b_{\mathrm{A}}(t)}= & \left(V_{\mathrm{A}}(t)-b_{\mathrm{A}}(t)\right) h\left(b_{\mathrm{A}}(t)\right) \\
& -\sigma\left(V_{\mathrm{B}}(t), b_{\mathrm{A}}(t)\right) h\left(b_{\mathrm{A}}(t)\right)=0,
\end{aligned}
$$

then:

$$
V_{\mathrm{A}}(t)-b_{\mathrm{A}}(t)-\sigma\left(V_{\mathrm{B}}(t), b_{\mathrm{A}}(t)\right)=0 .
$$

Hence, according to Proposition 1, we have $1+$ $\frac{\partial \sigma\left(v_{\mathrm{B}}(t), x\right)}{\partial x}>0$. Again, on the base of Corollary 2, $x \geq b_{\mathrm{A}}(t)$ in Eq. (8) means $t_{x} \leq t$. Moreover, $t_{x} \leq \theta \leq 1$ for $\forall \theta \in \Omega(x)=\left\{\theta \mid b_{\mathrm{A}}(\theta) \leq x\right\}$ while $x \geq b_{\mathrm{A}}(t)$.

$$
\begin{aligned}
\therefore & \Phi_{n-2 /-t}(z, \Omega(x))=P\left(\max _{\theta \in \Omega(x) \text { and } \theta \neq t} v_{\mathrm{B}}(\theta) \leq z\right) \\
& =\left(P \left(v_{\mathrm{B}}(\theta) \leq z \text { while } \theta \in \Omega(x)\right.\right. \\
& \left.\left.=\left\{\theta \mid b_{\mathrm{A}}(\theta) \leq x \cdot\right\} \text { and } t_{x} \leq \theta \leq 1\right)\right)^{n-2} \\
& =\left(\frac{\int_{1+\beta-z}^{1} f(\theta) d \theta}{\int_{t_{x}}^{1} f(\theta) d \theta}\right)^{n-2}=\left(\frac{z-\beta}{1-t_{x}}\right)^{n-2},
\end{aligned}
$$

where $z \geq V_{\mathrm{B}}(1)=1-(1-\beta)=\beta$ and $f(\theta)=1$ in $[0,1]$.

Next, we discuss the expected surplus in various cases as follows:

1. $\beta \in W 1$ :

$$
\begin{aligned}
& \sigma\left(v_{\mathrm{B}}(t), x\right)=\int_{0}^{v_{\mathrm{B}}(t)}\left(v_{\mathrm{B}}(t)-z\right) d \Phi_{n-2 /-t}(z, \Omega(x)) \\
& =\int_{\beta}^{1+\beta-t}(1+\beta-t-z) \frac{(n-2)}{1-t_{x}}\left(\frac{z-\beta}{1-t_{x}}\right)^{n-3} d z \\
& =\frac{(1-t)^{n-1}}{(n-1)\left(1-t_{x}\right)^{n-2}} .
\end{aligned}
$$

According to Corollary 2, $x=b_{\mathrm{A}}(t)$ means $t_{x}=t$.

$$
\text { Hence, } \sigma\left(v_{\mathrm{B}}(t), b_{\mathrm{A}}(t)\right)=\frac{1-t}{n-1} \text {; }
$$

2. $\beta \in W 2$ :

$$
\begin{aligned}
& \sigma\left(v_{\mathrm{B}}(t), x\right)=\int_{0}^{v_{\mathrm{B}}(t)}\left(v_{\mathrm{B}}(t)-z\right) d \Phi_{n-2 /-t}(z, \Omega(x)) \\
& =\int_{\beta}^{1-\beta+t}(1-\beta+t-z) \frac{(n-2)}{1-t_{x}}\left(\frac{z-\beta}{1-t_{x}}\right)^{n-3} d z \\
& =\frac{(1-2 \beta+t)^{n-1}}{(n-1)\left(1-t_{x}\right)^{n-2}} .
\end{aligned}
$$

Hence:

$$
\sigma\left(v_{\mathrm{B}}(t), b_{\mathrm{A}}(t)\right)=\frac{(1-2 \beta+t)^{n-1}}{(n-1)(1-t)^{n-2}} .
$$

3. $\beta \in W 3: \beta \geq(1+t) / 2$ means $v_{\mathrm{B}}(t)=1-\beta+t \leq \beta$.

Then:

$$
\begin{array}{r}
\sigma\left(v_{\mathrm{B}}(t), x\right)=\int_{\beta}^{v_{\mathrm{B}}(t)}\left(v_{\mathrm{B}}(t)-z\right) \\
d \Phi_{n-2 /-t}(z, \Omega(x))=0 .
\end{array}
$$

Hence:

$$
\sigma\left(v_{\mathrm{B}}(t), b_{\mathrm{A}}(t)\right)=0 .
$$

Therefore, Bidder $t$ 's equilibrium bid for Item A should be $b_{\mathrm{A}}(t)=1-t-\sigma\left(v_{\mathrm{B}}(t), b_{\mathrm{A}}(t)\right)$, where $\sigma\left(v_{\mathrm{B}}(t), b_{\mathrm{A}}(t)\right)$ is shown as in Eqs. (5), (6), and (7).

It is also easy to verify $\frac{d b_{\mathrm{A}}(t)}{d t}<0$ through Eqs. (5), (6), and (7). Thus, Item A will be allocated to the bidder with the highest valuation, namely one closest to A.

Remark 5. Eqs. (5), (6), and (7) imply that Bidder $t=1$ or $t \leq 2 \beta-1$ always bids his or her true valuation in the first stage because he or she as a preponderant rival of Item A believes in advance that his or her expected surplus of winning Item B will be 0 . However, Bidders $t=0$ always hide their surplus $\frac{(1-2 \max \{\beta, 1 / 2\})^{n-1}}{(n-1)}$ in the first stage by making use of the advantage of their location.

\subsection{Equilibrium bids when hiding $\beta$}

Item $\mathrm{B}$ (namely $\beta$ ) is hidden before the end of auctioning $\mathrm{A}$. Each bidder knows $\alpha=0$, his or her own location (or type) $t$, and bidders' number $n$ and does not know $\beta$ and other bidders' locations (or types). However, he or she regards other bidders' locations as random variables distributed in $[0,1]$, and believes that Item B is also distributed in $[0,1]$. Similar to Section 4.1 , the 
key to determining the equilibrium bid is the expected surplus of winning Item B.

While $\beta$ is regarded as a random variable, Bidder $t$ 's expected surplus of winning $\mathrm{B}$ is $\sigma\left(\cdot, b_{\mathrm{A}}^{\prime}(t)\right)=$ $E_{\beta}\left[\sigma\left(v_{\mathrm{B}}(t), b_{\mathrm{A}}(t)\right)\right]$, and Bidder $t$ 's equilibrium bid for Item A should be $b_{\mathrm{A}}^{\prime}(t)=v_{\mathrm{A}}(t)-\sigma\left(\cdot, b_{\mathrm{A}}^{\prime}(t)\right)$. Hence:

$$
\begin{aligned}
\sigma\left(\cdot, b_{\mathrm{A}}^{\prime}(t)\right)=E_{\beta}\left[\sigma\left(v_{\mathrm{B}}(t), b_{\mathrm{A}}(t)\right)\right] \\
=\int_{0}^{t} \sigma\left(v_{\mathrm{B}}(t), b_{\mathrm{A}}(t)\right) g(\beta) d \beta=\int_{0}^{t} \frac{1-t}{n-1} d \beta \\
\quad+\int_{t}^{\frac{1+t}{2}} \frac{(1-2 \beta+t)^{n-1}}{(n-1)(1-t)^{n-2}} d \beta \\
=\frac{(1-t)(1+(2 n-1) t)}{2 n(n-1)} .
\end{aligned}
$$

Proposition 5. If $\beta$ is hidden before the end of auctioning Item $\mathrm{A}$, then Bidder $t$ 's equilibrium bid for Item $\mathrm{A}$ is $b_{\mathrm{A}}^{\prime}(t)=1-t-\sigma\left(\cdot, b_{\mathrm{A}}^{\prime}(t)\right)$, where $\sigma\left(\cdot, b_{\mathrm{A}}^{\prime}(t)\right)$ is introduced in Eq. (9).

Proof of Proposition 5 is similar to that of Proposition 4. Further:

$$
\begin{aligned}
\frac{d b_{\mathrm{A}}^{\prime}(t)}{d t} & =\frac{d}{d t}\left[1-t-\left(\frac{1+2(n-1) t-(2 n-1) t^{2}}{2 n(n-1)}\right)\right] \\
& =\frac{2-n}{n-1}<0(\because n \geq 3) .
\end{aligned}
$$

Thus, $b_{\mathrm{A}}^{\prime}(t)$ is monotonically decreasing with $t$, which is consistent with Proposition 2. Concurrently, $\frac{d b_{\mathrm{A}}^{\prime}(t)}{d t}<$ 0 also indicates that Item $\mathrm{A}$ will be allocated to the bidder with the highest valuation, namely one closest to Item A.

Remark 6. According to Eq. (9), $\sigma\left(\cdot, b_{\mathrm{A}}^{\prime}(0)\right)=$ $\frac{1}{2 n(n-1)}, \sigma\left(\cdot, b_{\mathrm{A}}^{\prime}(0.5)\right)=\frac{2 n+1}{8 n(n-1)}$, and $\sigma\left(\cdot, b_{\mathrm{A}}^{\prime}(1)\right)=0$. It can easily be proved that $\sigma\left(\cdot, b_{\mathrm{A}}^{\prime}(t)\right)$ reaches the maximum $\frac{n}{2(n-1)(2 n-1)}$ while $t=\frac{n-1}{2 n-1}$, and $\sigma\left(\cdot, b_{\mathrm{A}}^{\prime}(t)\right)$ is monotonically increasing with $t$ in $\left[0, \frac{n-1}{2 n-1}\right]$ and monotonically decreasing in $\left[\frac{n-1}{2 n-1}, 1\right]$. Thus, bidders in $\left[0, \frac{n-1}{2 n-1}\right]$ have advantages over those in $\left[\frac{n-1}{2 n-1}, 1\right]$.

\subsection{Further researches}

Let $R_{k}(n)$ denote the auctioneer's expected revenue from selling Item $k \in\{\mathrm{A}, \mathrm{B}\}$ while $\beta$ is revealed and $R_{k}^{\prime}(n)$ denote the aforementioned expected revenue while $\beta$ is hidden, where $n$ is the number of bidders. Then, $R(n)=R_{\mathrm{A}}(n)+R_{\mathrm{B}}(n)$ is the auctioneer's total expected revenue at two auctions while $\beta$ is revealed and $R^{\prime}(n)=R_{\mathrm{A}}^{\prime}(n)+R_{\mathrm{B}}^{\prime}(n)$ is the aforementioned total revenue while $\beta$ is hidden. In the second stage, the dominant strategies of bidders are to bid their own valuations. Accordingly, expected revenues from selling Item $\mathrm{B}$ are the same under the above two information policies, namely $R_{\mathrm{B}}(n)=R_{\mathrm{B}}^{\prime}(n)$. Thus, the difference between the total expected revenues $R(n)$ and $R^{\prime}(n)$ is mainly determined by $R_{\mathrm{A}}(n)$ and $R_{\mathrm{A}}^{\prime}(n)$.

Different from bidders, an auctioneer only knows $\alpha, \beta$, and $n$ in advance and does not know locations of bidders, yet believes that bidders are distributed in $[0,1]$. Therefore, with respect to the expected revenue from selling Item $\mathrm{A}, R_{\mathrm{A}}(n)$ or $R_{\mathrm{A}}^{\prime}(n)$ is determined by the expectation of $\max _{t} b_{\mathrm{A}}(t)$ or $\max _{t} b_{\mathrm{A}}^{\prime}(t)$. Thus, while $F(t)=t$ or $f(t)=1$ in $[0,1]$ :

$$
\begin{aligned}
& R_{\mathrm{A}}(n)=\int_{0}^{1}\left(1-t-\sigma\left(v_{\mathrm{B}}(t), b_{\mathrm{A}}(t)\right)\right) \cdot n(1-F(t))^{n-1} d t \\
& =\int_{0}^{1}(1-t) \cdot n(1-t)^{n-1} d t \\
& -\left[\int_{\max \{2 \beta-1,0\}}^{\beta} \frac{(1-2 \beta+t)^{n-1}}{(n-1)(1-t)^{n-2}} \cdot n(1-t)^{n-1} d t\right. \\
& \left.+\int_{\beta}^{1} \frac{1-t}{n-1} \cdot n(1-t)^{n-1} d t\right] \\
& =\left\{\begin{array}{cc}
1-\frac{1}{n+1}-\frac{2}{n-1}(1-\beta)^{n+1} & \\
+\frac{(n+2-2 \beta)(1-2 \beta)^{n}}{(n+1)(n-1)} & \text { for } \beta \in\left[0, \frac{1}{2}\right] \\
1-\frac{1}{n+1}-\frac{2}{n-1}(1-\beta)^{n+1} & \text { for } \beta \in\left[\frac{1}{2}, 1\right]
\end{array}\right. \\
& R_{\mathrm{A}}^{\prime}(n)=\int_{0}^{1}\left(1-t-\sigma\left(\cdot, b_{\mathrm{A}}^{\prime}(t)\right)\right) \cdot n(1-F(t))^{n-1} d t \\
& =\int_{0}^{1}\left[(1-t)-\frac{1+2(n-1) t-(2 n-1) t^{2}}{2 n(n-1)}\right] \\
& \cdot n(1-t)^{n-1} d t=1-\frac{(2 n-1)(n+3)}{2(n-1)(n+1)(n+2)} \text {. }
\end{aligned}
$$

Curve clusters of $R_{\mathrm{A}}(n)$ and $R_{\mathrm{A}}^{\prime}(n)$ with $n$ and $\beta$ are shown in Figures 3 and 4, respectively. They visually show the following propositions.

Proposition 6. $R_{\mathrm{A}}(n)$ is monotonically increasing functions of $n$ and $\beta ; R_{\mathrm{A}}^{\prime}(n)$ is monotonically increasing functions of $n$. 


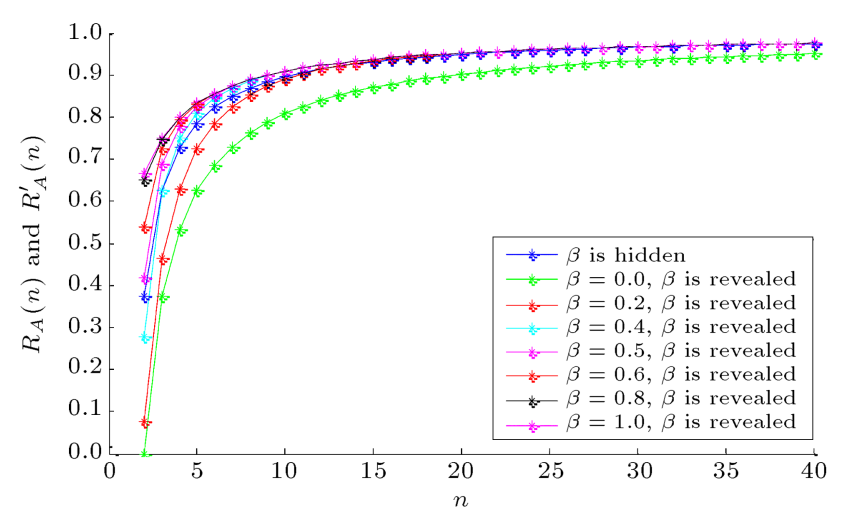

Figure 3. Curve clusters of $R_{\mathrm{A}}(n)$ and $R_{\mathrm{A}}^{\prime}(n)$ as functions of $n$.

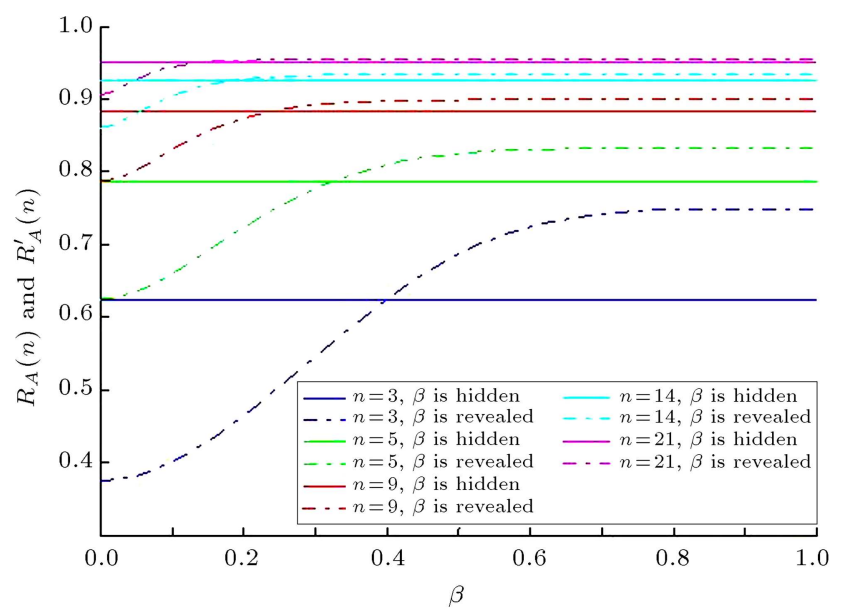

Figure 4. Curve clusters of $R_{\mathrm{A}}(n)$ and $R_{\mathrm{A}}^{\prime}(n)$ as functions of $\beta$.

\section{Proof.}

1. While $\beta \in\left[\frac{1}{2}, 1\right], R_{\mathrm{A}}(n)=1-\frac{1}{n+1}-\frac{2}{n-1}(1-\beta)^{n+1}$. Obviously, $R_{\mathrm{A}}(n)$ is monotonically increasing functions of $n$ and $\beta$.

While $\beta \in\left[0, \frac{1}{2}\right]$ :

$$
\begin{aligned}
R_{\mathrm{A}}(n)= & -\frac{1}{n+1}-\frac{2}{n-1}(1-\beta)^{n+1} \\
& +\frac{(n+2-2 \beta)(1-2 \beta)^{n}}{(n+1)(n-1)} .
\end{aligned}
$$

Then:

$$
\begin{aligned}
& \frac{d R_{\mathrm{A}}(n)}{d \beta}=\frac{2(n+1)}{n-1}(1-\beta)^{n} \\
& \quad-\frac{2(1-2 \beta)^{n}+2 n(n+2-2 \beta)(1-2 \beta)^{n-1}}{(n+1)(n-1)} \\
& \quad=\frac{2}{n-1}\left[(n+1)(1-\beta)^{n}-n(1-2 \beta)^{n-1}\right.
\end{aligned}
$$

$$
\begin{aligned}
& \left.-(1-2 \beta)^{n-1}\right]=\frac{2}{n-1}\left[n \left((1-\beta)^{n}\right.\right. \\
& \left.\left.-(1-2 \beta)^{n-1}\right)+\left((1-\beta)^{n}-(1-2 \beta)^{n}\right)\right] \\
& =\frac{2}{n-1}\left[n\left((1-\beta)^{n}-(1-2 \beta)^{n-1}\right)\right. \\
& \left.+\left((1-\beta)^{n}-(1-2 \beta)^{n}\right)\right],
\end{aligned}
$$

$\because \beta \in\left[0, \frac{1}{2}\right], \therefore 1-\beta \geq 1-2 \beta \geq 0$ and $(1-\beta)^{2}=$ $1-2 \beta+\beta^{2} \geq 1-2 \beta \geq 0$. Hence, $(1-\beta)^{n} \geq(1-2 \beta)^{n}$ and $(1-\beta)^{n} \geq(1-2 \beta)^{n-1}$. Therefore, $\frac{d R_{\mathrm{A}}(n)}{d \beta} \geq 0$, namely $R_{\mathrm{A}}(n)$ is monotonically increasing functions of $\beta$.

Again, according to Eq. (9), let:

$$
\begin{aligned}
R_{\mathrm{A}}(x) & =\int_{0}^{1} x(1-t)^{x} d t \\
& -\left[\int_{0}^{\beta} \frac{x}{(x-1)}(1-2 \beta+t)^{x-1}(1-t) d t\right. \\
& \left.+\int_{\beta}^{1} \frac{x}{x-1}(1-t)^{x} d t\right] \\
& =\frac{x}{x+1}-\left[\int_{0}^{\beta} h_{1}(x) d t+\int_{\beta}^{1} h_{2}(x) d t\right] .
\end{aligned}
$$

Then:

$$
\begin{aligned}
& \frac{d h_{1}(x)}{d x}=\frac{d}{d x}\left(\frac{x}{(x-1)}(1-2 \beta+t)^{x-1}(1-t)\right) \\
& =\frac{(1-2 \beta+t)^{x-1}(1-t)}{(x-1)^{2}}(x(x-1) \ln (1-2 \beta+t)-1) \\
& \leq 0 \quad(\because x \geq 3 \text { and } 0 \leq 1-2 \beta+t \leq 1),
\end{aligned}
$$

$$
\begin{aligned}
& \frac{d h_{2}(x)}{d x}=\frac{d}{d x}\left(\frac{x}{x-1}(1-t)^{x}\right) \\
& \quad=\frac{(1-t)^{x}}{(x-1)^{2}}(x(x-1) \ln (1-t)-1) \leq 0
\end{aligned}
$$$$
\therefore \frac{\partial R_{\mathrm{A}}(x)}{\partial x}=\frac{1}{(x+1)^{2}}-\left[\int_{0}^{\beta} \frac{d h_{1}(x)}{d x} d t+\int_{\beta}^{1} \frac{d h_{2}(x)}{d x} d t\right]
$$

$>0$.

Hence, $R_{\mathrm{A}}(n)$ or $R_{\mathrm{A}}(x)$ is monotonically increasing functions of $n$ or $x$; 
2. $R_{\mathrm{A}}^{\prime}(n)=1-\frac{(2 n-1)(n+3)}{2(n-1)(n+1)(n+2)}=1-\frac{n-2}{n^{2}-1}-$ $\frac{5}{2(n-1)(n+2)}$. Obviously, $R_{\mathrm{A}}^{\prime}(n)$ is monotonically increasing functions of $n$, owing to $n \geq 3$.

Eqs. (10) and (11) show complicated influences of $\beta$ and $n$ on the expected revenue. Here, both Proposition 6 and Figure 4 imply that if $n$ is given, then there must exist $\beta_{0}$ to satisfy $R_{\mathrm{A}}(n) \leq R_{\mathrm{A}}^{\prime}(n)$ while $\beta \leq \beta_{0}$, and $R_{\mathrm{A}}(n) \geq R_{\mathrm{A}}^{\prime}(n)$ while $\beta \geq \beta_{0}$. However, it is difficult to fix the exact value of $\beta_{0}$ with $n$. Next, Proposition 7 provides some sufficient conditions with respect to comparisons of both $R_{\mathrm{A}}(n)$ and $R_{\mathrm{A}}^{\prime}(n)$.

Proposition 7. Let $n \geq 3$.

1. $R_{\mathrm{A}}(n) \leq R_{\mathrm{A}}^{\prime}(n)$ while $\beta \leq 1-\left(\frac{3 n+1}{2 n(n+2)}\right)^{\frac{1}{n+1}}$;

2. $R_{\mathrm{A}}(n)>R_{\mathrm{A}}^{\prime}(n)$ while $\beta \geq 1-\left(\frac{3 n+1}{4(n+1)(n+2)}\right)^{\frac{1}{n+1}}$.

\section{Proof.}

1. While $\beta \leq 1-\left(\frac{3 n+1}{2 n(n+2)}\right)^{\frac{1}{n+1}},(1-\beta)^{n+1} \geq \frac{3 n+1}{2 n(n+2)}$. $\because(1-\beta)^{2} \geq 1-2 \beta, \therefore \frac{(n+2-2 \beta)}{(n+1)(n-1)}(1-2 \beta)^{n} \leq$ $\frac{(n+2)}{(n+1)(n-1)}(1-\beta)^{n+1}$. Hence:

$$
\begin{aligned}
(1 & -\frac{1}{n+1}-\frac{2}{n-1}(1-\beta)^{n+1} \\
& \left.+\frac{(n+2-2 \beta)(1-2 \beta)^{n}}{(n+1)(n-1)}\right) \\
& -\left(1-\frac{(2 n-1)(n+3)}{2(n-1)(n+1)(n+2)}\right) \leq 0 .
\end{aligned}
$$

Inequation (12) means $R_{\mathrm{A}}(n) \leq R_{\mathrm{A}}^{\prime}(n)$ for $\beta \in$ $\left[0, \frac{1}{2}\right]$ and, also, means $R_{\mathrm{A}}(n) \leq R_{\mathrm{A}}^{\prime}(n)$ for $\beta \in$ $\left[\frac{1}{2}, 1\right]$.

Hence, according to Proposition $6, R_{\mathrm{A}}(n) \leq$ $R_{\mathrm{A}}^{\prime}(n)$ for $\beta \in[0,1]$.

2. While $\beta \geq 1-\left(\frac{3 n+1}{4(n+1)(n+2)}\right)^{\frac{1}{n+1}},(1-\beta)^{n+1} \leq$ $\frac{3 n+1}{4(n+1)(n+2)}$. Then:

$$
\begin{aligned}
& \left(1-\frac{1}{n+1}-\frac{2}{n-1}(1-\beta)^{n+1}\right) \\
& \quad-\left(1-\frac{(2 n-1)(n+3)}{2(n-1)(n+1)(n+2)}\right) \geq 0 .
\end{aligned}
$$

Inequation (13) means $R_{\mathrm{A}}(n) \geq R_{\mathrm{A}}^{\prime}(n)$ for $\beta \in\left[\frac{1}{2}, 1\right]$. $\because \frac{(n+2-2 \beta)(1-2 \beta)^{n}}{(n+1)(n-1)} \geq 0, \therefore$

Inequation (13) also means $R_{\mathrm{A}}(n) \geq R_{\mathrm{A}}^{\prime}(n)$ for $\beta \in\left[0, \frac{1}{2}\right]$.
Hence, according to Proposition $6, R_{\mathrm{A}}(n) \geq$ $R_{\mathrm{A}}^{\prime}(n)$ for $\beta \in[0,1]$.

Here, $R_{\mathrm{A}}(n) \leq R_{\mathrm{A}}^{\prime}(n)\left(R_{\mathrm{A}}(n)>R_{\mathrm{A}}^{\prime}(n)\right)$ means that expected revenue for $\mathrm{A}$ while revealing $\beta$ is less (more) than that while hiding $\beta$. Proposition 7 implies that revealing the latter items in advance would uncertainly affect the overall efficiency and revenues of sequential auctions, which is consistent with findings of Cason [5], Jane and David [13], Mikusheva [9], Jackson and Kremer [10], Kannan [11], Rao et al. [16,17], and Colucci et al. [18]. Owing to $R_{\mathrm{B}}(n)=R_{\mathrm{B}}^{\prime}(n)$, Proposition 7 is also sufficient to compare $R(n)$ with $R^{\prime}(n)$.

\section{Conclusions}

Focusing on sequential auctions of close substitutes with slightly more general associated valuations, this paper constructed a class Hotelling model and discussed equilibrium bids under second-price sealed-bid auction formats. Conclusions showed that sequential auctions described by this model were efficient while bidders' valuations satisfied conditions given by Corollary 2. Thus, the class Hotelling model could be used as a support to deal with some auctions in supply chains. It is helpful for the analysis and design of some business mechanisms.

Through the instrumentality of this model, some sequential auctions were specifically explored, while a bidder's valuation was a linear function of a distance between him or her and an item. The equilibrium bid of a bidder as a preponderant rival was deduced and verified. In addition, it depends on both numbers of bidders and locations of items whether the latter item (namely Item B) should be revealed or hidden. Generally, revealing information usually improves revenues of auctions with assumptions of independent valuations for multi-items. However, our conclusions are more complicated because each bidder's valuations for Items $\mathrm{A}$ and $\mathrm{B}$ are not independent in our paper, yet are correlated. In this paper, although the sequential auctions with only two items seem simple or farfetched, the characterization of the class Hotelling model for two items is an important step in achieving similar characterizations of models with more than two items.

\section{Acknowledgements}

We would like to thank anonymous referees for useful comments. This work is supported by the National Natural Science Foundation of China (Nos. 71471069, 71671135), the 2018 Soft Science Research Project of Technology Innovation in Hubei province (No. 2018ADC044), the Humanities and Social Science Research Project of Hubei Province Department of Ed- 
ucation(No. 17Q068), and the Fundamental Research Funds for the Central Universities (WUT: 2019IB013). We thank them for their various contributions to this work, too.

\section{References}

1. Osborne, M.J. and Pitchik, C. "Equilibrium in Hotelling's model of spatial competition", Econometrica, 55(4), pp. 911-922 (1987).

2. Takatoshi, T. "Multiproduct firms in Hotelling's spatial competition", Journal of Economics \& Management Strategy, 21(2), pp. 445-467 (2012).

3. Peter, F. "Auctions with interdependent valuations", International Journal of Game Theory, 25, pp. 51-64 (1996).

4. Milgrom, P. and Weber, R. "A theory of auctions and competitive bidding", Econometrica, 50(5), pp. 10891122 (1982).

5. Cason, T.N. "An experimental study of information revelation policies in sequential auctions", Management Science, 57(4), pp. 667-688 (2011).

6. Benoît, J.P. and Dubra, J. "Information revelation in auctions", Games and Economics Behavior, 57(2), pp. 181-205 (2006).

7. Silva, D., Dunne, T., Kankanamge, A., and Kosmopoulou, G. "The impact of public Iinformation on bidding in highway procurement auctions", European Economic Review, 52(1), pp. 150-181 (2008).

8. Szech, N. "Optimal disclosure of costly information packages in auctions", Journal of Mathematical Economics, 47(4-5), pp. 462-469 (2011).

9. Mikusheva, A. and Sonin, K., Information revelation and efficiency in auctions, CEPR Discussion Paper (2002).

10. Jackson, M. and Kremer, I. "The relationship between the allocation of goods and a seller's revenue", Journal of Mathematical Economics, 40, pp. 371-392 (2004).

11. Kannan, K.N. "Effects of information revelation policies under cost uncertainty", Information Systems Research, 23(1), pp. 75-92 (2013).

12. Mezzetti, C. and Tsetlin, I. "On the lowest-winningbid and the highest-Losing-Bid auctions", Journal of Mathematical Economics, 44, pp. 1040-1048 (2008).
13. Black, J. and Meza, D.D. "Systematic price differences between successive auctions are no anomaly", Journal of Economics \& Management Strategy, 1(4), pp. 607628 (1992).

14. Zeithammer, R., Sequential Auctions with Information about Future Goods, Research Paper (2010).

15. Thomas, C.J. "Information revelation and buyer profits in repeated procurement competition", The Journal of Industrial Economics, 58(1), pp. 79-105 (2010).

16. Rao, C.J., Xiao, X.P., Goh, M., Zheng, J.J., and Wen, J.H. "Compound mechanism design of supplier selection based on multi-attribute auction and risk management of supply chain", Computers \& Industrial Engineering, 105, pp. 63-75 (2017).

17. Rao, C.J., Goh, M., Zhao, Y., and Zheng, J.J. "Location selection of sustainability city logistics centers", Transportation Research Part D: Transport and Environment, 36, pp. 29-44 (2015).

18. Colucci, D., Doni, N., and Valori, V. "Preferential treatment in procurement auctions through information revelation", Economics Letters, 117(3), pp. 883886 (2012).

\section{Biographies}

Erqin Hu received an MS degree from Huazhong University of Science and Technology, China in 2005 and received her $\mathrm{PhD}$ degree from Huazhong University of Science and Technology, China in 2017. Her research interests include auction theory and decision theory and method.

Congjun Rao received an MS degree from Wuhan University of Technology, China in 2006 and received his $\mathrm{PhD}$ degree from Huazhong University of Science and Technology, China in 2011. His research interests include supply chain management, auction theory, and decision theory and method.

Yong Zhao is currently a Professor at the Department of Control Science and Engineering of Huazhong University of Science and Technology. His BSc, MSc, and $\mathrm{PhD}$ degree were obtained from Huazhong University of Science and Technology in Systems Engineering. His main research interests include decision theory and methods, engineering economics, and auction theory. 